\title{
Spinal Cord Ischemia Secondary to Hypovolemic Shock
}

\author{
Jacob YL Oh, Siddhant Kapoor, Roy KM Koh, Eugene WR Yang, Hwan-Tak Hee \\ Department of Orthopaedic Surgery, Khoo Teck Puat Hospital, Singapore
}

\begin{abstract}
A 44-year-old male presented with symptoms of spinal cord compression secondary to metastatic prostate cancer. An urgent decompression at the cervical-thoracic region was performed, and there were no complications intraoperatively. Three hours postoperatively, the patient developed acute bilateral lower-limb paralysis (motor grade 0). Clinically, he was in class 3 hypovolemic shock. An urgent magnetic resonance imaging (MRI) was performed, showing no epidural hematoma. He was managed aggressively with medical therapy to improve his spinal cord perfusion. The patient improved significantly, and after one week, he was able to regain most of his motor functions. Although not commonly reported, spinal cord ischemia post-surgery should be recognized early, especially in the presence of hypovolemic shock. MRI should be performed to exclude other potential causes of compression. Spinal cord ischemia needs to be managed aggressively with medical treatment to improve spinal cord perfusion. The prognosis depends on the severity of deficits, and is usually favorable.
\end{abstract}

Keywords: Spinal cord ischemia; Hypovolemic shock

\section{Introduction}

Spinal cord ischemia is commonly seen after aortic procedures, with a prevalence rate ranging $3 \%-14 \%$ [1]. Other associated causes include atherosclerosis, cardiac embolism, epidural anesthesia and hypotension-induced surgery $[2,3]$. We report a rare case of postoperative spinal cord ischemia following a spinal surgery. This is a critical postsurgery neurological complication, which is reversible in nature. In this case report, we highlight the importance of early diagnosis and suggest a management approach. With the appropriate treatment, the outcome can be favorable.

\section{Case Report}

A 44-year-old man with a medical history of hyperten- sion and diabetes presented with a 3-month duration of progressive upper back pain and myelopathic symptoms. His chief complaint was unsteadiness with ambulation. In addition, he also noticed increasing hand numbness and weakness in his grip strength.

Clinical examination revealed tenderness at the midthoracic region. He was unable to walk without aid, due to poor balance. The power of his upper limbs was weak, with severe wasting of his triceps and intrinsic muscles of the hand (Medical Research Council, grade 4 power for C5 and C6; grade 3 for C7, C8, and T1 bilaterally). The power in his lower limbs was full. Sensation was preserved; and there was no hyper-reflexia, clonus or spasticity. The Hoffman's test was negative.

A magnetic resonance imaging (MRI) revealed that this sclerotic lesion had infiltrated the spinal canal, and

Received Nov 24, 2013; Revised Jan 9, 2014; Accepted Jan 24, 2014

Corresponding author: Jacob YL Oh

Department of Orthopaedic Surgery, Khoo Teck Puat Hospital, 90 Yishun Central, Singapore 768828

Tel: +65-9155-5918, E-mail: Jacob_oh@yahoo.com 
was causing significant spinal cord compression. In addition, there were other lesions in the cervical spine at C5 and C6, which showed cord compression with spinal cord signal changes (Fig.1A, B). Radiographs of the cervical spine were unremarkable, but the thoracic spine showed a sclerotic lesion at T5 (Fig.1C, D).

Blood investigation showed normal infective and inflammatory markers (white blood cell, 9.02; erythrocyte sedimentation rate, 6; C-reactive protein, 1.9), with an abnormally high prostate-specific antigen (PSA $>100)$. A Tc99 bone scan showed vertebral hotspots similar to those identified on the MRI. There were no other lesions
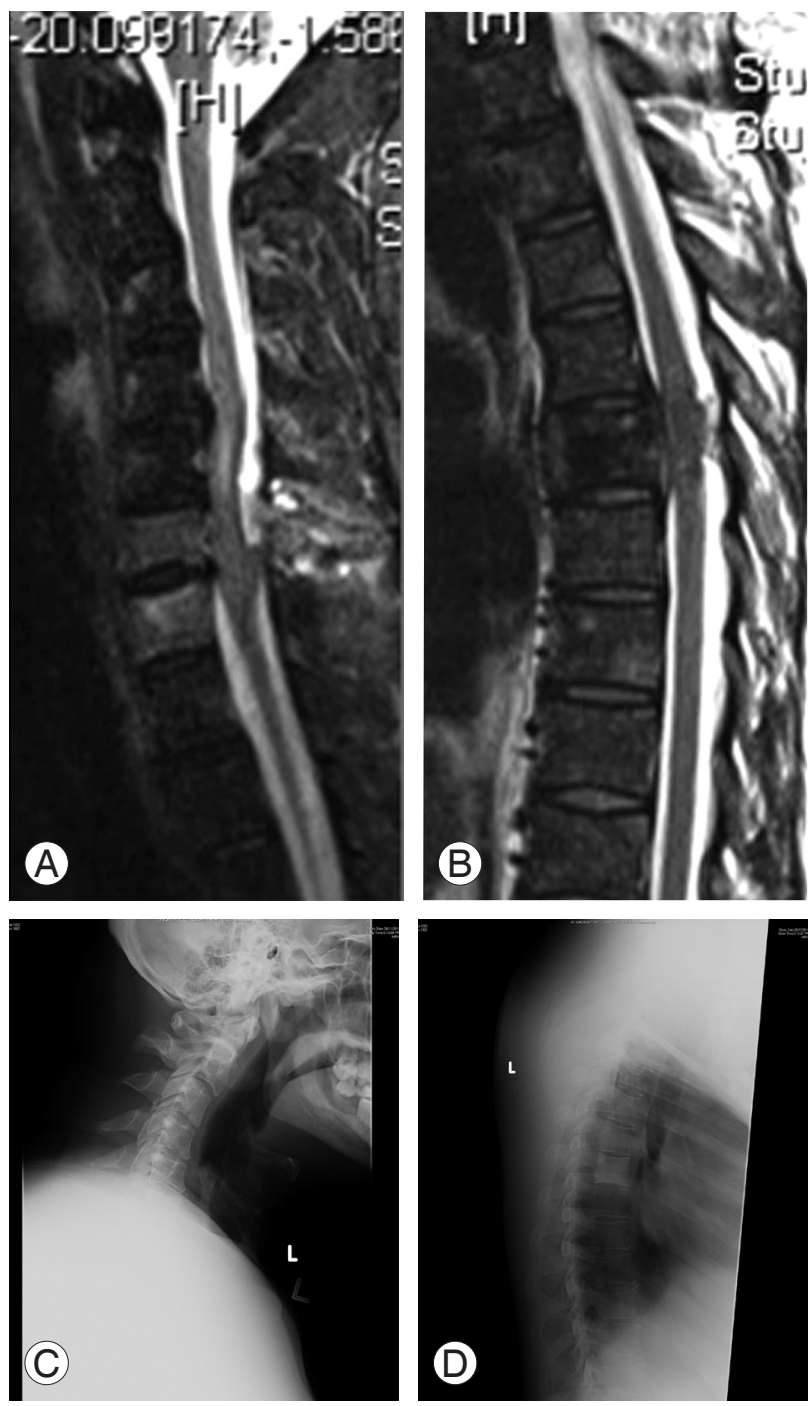

Fig. 1. (A) Magnetic resonance cervical spine showing high intensity at the $\mathrm{C} 5$ and $\mathrm{C} 6$ vertebral bodies. There is an associated cord compression with cord signal changes at the level above. (B) Thoracic lesion at T5 causing cord compression. (C) Lateral cervical. (D) Lateral thoracic spine preoperative radiograph showing sclerotic lesion at T5. apart from the multiple ribs involvement $(>3)$. CT scan revealed an isolated lung lesion and an enlarged prostate. No liver metastasis was found. A TRUS (transurethral biopsy) performed by the urologist returned with a histology that was consistent with prostatic adenocarcinoma. The patient was diagnosed with stage 4 prostate cancer, with metastasis to the spine, presenting as cord compression. He was started on dexamethasone and scheduled for surgical decompression and stabilization. His preoperative hemoglobin was $16.7 \mathrm{~g} / \mathrm{L}$.

A posterior decompression (laminectomy) of C5, C6, and $\mathrm{T} 5$ with an instrumented fusion from $\mathrm{C} 3-\mathrm{T} 3$ was performed. The procedure was uncomplicated. His intraoperative blood pressure was stable around 90/60 mm $\mathrm{Hg}$, with no hypotensive episodes. His heart rate never exceeded 110 beats per minute. Blood pressure was closely monitored using a combination of arterial and noninvasive blood pressure measurements. The surgery took a total of 4 hours and 10 minutes. Throughout the surgery, there was no drop in the neuro-monitoring signals (sensory/motor-evoked potentials). Rather, the sensory signals (amplitude) for the right lower limbs improved following the decompression.

There was an estimated intra-operative blood loss of $3.0 \mathrm{~L}$, with the majority arising from the decompression stage of the surgery. An intraoperative transfusion of 4 units pack cells (approximately $1 \mathrm{~L}$ ), $1.5 \mathrm{~L}$ of Ringer's lactate and 1.0 $\mathrm{L}$ of colloid were transfused. Once the patient was extubated, he was reviewed in the high dependency ward. He was alert, moving his legs with lower limb power of grade 5. There was no change in the power of his upper limbs.

Three hours postoperatively, the patient complained that he was unable to move both his legs. Clinical examination revealed motor power of grade 0 bilaterally from L2 to S1. Sensation of all dermatomes was preserved, and his anal tone was intact. Vital signs at this time showed tachycardia of up to 130 beats per minute, and persistent hypotension with a systolic blood pressure of $<80 \mathrm{~mm}$ Hg. He was still alert and not confused. His drain output at this time was $1,000 \mathrm{~mL}$. His hemoglobin was $9.4 \mathrm{~g} /$ $\mathrm{L}$, indicating a drop of $7.2 \mathrm{~g} / \mathrm{L}$ from his preoperative hemoglobin of $16.7 \mathrm{~g} / \mathrm{L}$. An urgent MRI scan was ordered to exclude the possibility of hematoma causing extrinsic cord compression. However, there was no sign of external compression. Instead, an improved canal diameter following the decompression was seen (Fig. 2A, B). The implants were well-positioned (Fig. 2C, D). 
A diagnosis of hypotensive shock causing spinal cord ischemia was made. As there was no extrinsic compression observed, a medical treatment to improve his cord perfusion was initiated. This included a blood transfusion to keep his hemoglobin higher than $>10 \mathrm{~g} / \mathrm{L}$, nasal oxygen and maintaining a normotensive blood pressure.
Dexamethasone $8 \mathrm{mg}$, three times a day, started preoperatively was continued. Over the next few days, the patient made good progress. The power in his lower limbs gradually improved from grade 0 to grade 4 over one week, with an improvement of one grade per day. By the end of two weeks, he was attempting to stand with assistance.
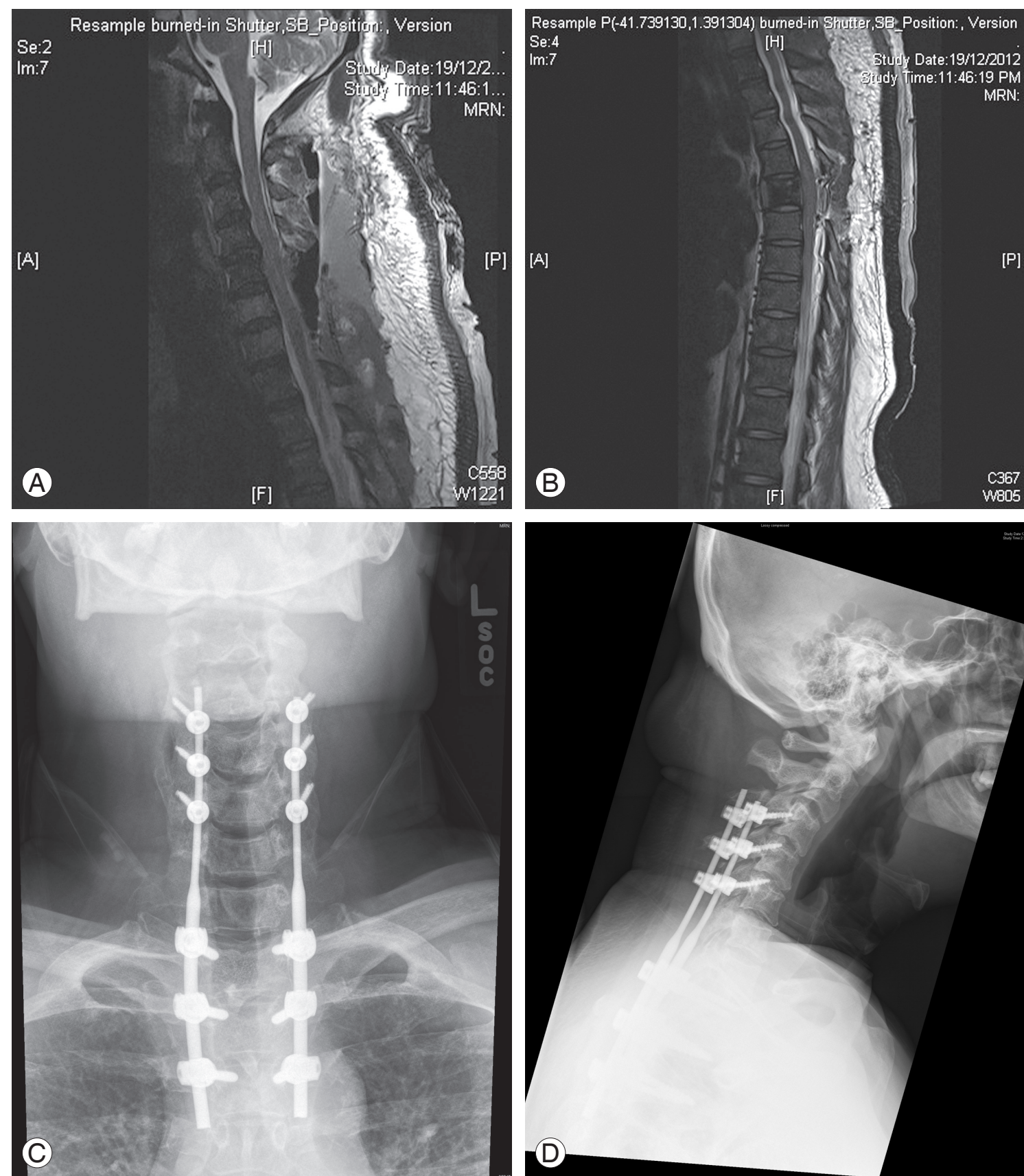

Fig. 2. (A, B) Immediate postoperative magnetic resonance showing no obvious extrinsic compression on the spinal cord (cervical and thoracic). (C) Postoperative lateral radiographs showing instrumentation from C3-T3. (D) Postoperative lateral radiographs showing instrumentation from C3-T3. 


\section{Discussion}

Spinal cord ischemia is commonly seen following aortic procedures, with a prevalence rate ranging $3 \%-14 \%$ [1]. Other associated causes include atherosclerosis, cardiac embolism, epidural anesthesia and even hypotension-induced surgery $[2,3]$. This is the first case reported after a spinal surgery, which we believe may be under-reported.

Spinal cord ischemia is a diagnosis of exclusion. A high index of suspicion is required, especially since the MRI scan may be normal in the acute phase. Alblas et al. [4] reported that the focal cord swelling and 'pencil-like' hyper-intensities which are typical of spinal cord ischemia manifest after 1-2 days of initial insult.

Our patient was diagnosed with spinal cord ischemia, having excluded all other possible causes. The sudden deterioration of power in his lower limbs was unlikely to be caused by an intraoperative injury, as the neuromonitoring signals remained constant throughout the surgery. Furthermore, he was able to move his limbs with motor strength of grade 5 once he was extubated. An urgent MRI helped us to rule out the possibility of hematoma causing delayed spinal cord compression. His final diagnosis of spinal cord ischemia also correlated with his clinical manifestation of hypotensive shock.

The outcome of spinal cord ischemia from most studies are favorable $[1,2,5]$, as is in this case. Ullery et al. [5] reported a series of twelve patients who developed spinal cord ischemia following aortic repair, of which nine (75\%) had complete neurological recovery. Nedeltchev et al. [2] found similar results, with a $71 \%$ return to ambulation in patients who had paraparesis. The authors however noted that the more severe the neurological deficit, the poorer the outcome (American Spinal Injury Association A, B) $[2,5]$.
In conclusion, spinal cord ischemia post-surgery should be recognized early, especially in the presence of hypovolemic shock. MRI should be performed to exclude other potential causes of compression such as a hematoma. Spinal cord ischemia should be managed aggressively with medical treatment to improve spinal cord perfusion. The prognosis depends on the severity of deficits, and is usually favorable.

\section{Conflict of Interest}

No potential conflict of interest relevant to this article was reported.

\section{References}

1. Maeda T, Yoshitani K, Sato S, et al. Spinal cord ischemia after endovascular aortic repair versus open surgical repair for descending thoracic and thoracoabdominal aortic aneurism. J Anesth 2012;26:80511.

2. Nedeltchev K, Loher TJ, Stepper F, et al. Long-term outcome of acute spinal cord ischemia syndrome. Stroke 2004;35:560-5.

3. Drummond JC, Lee RR, Owens EL. Spinal cord ischemia occurring in association with induced hypotension for colonic surgery. Anesth Analg 2012;114:1297-300.

4. Alblas CL, Bouvy WH, Lycklama A Nijeholt GJ, Boiten J. Acute spinal-cord ischemia: evolution of MRI findings. J Clin Neurol 2012;8:218-23.

5. Ullery BW, Cheung AT, Fairman RM, et al. Risk factors, outcomes, and clinical manifestations of spinal cord ischemia following thoracic endovascular aortic repair. J Vasc Surg 2011;54:677-84. 